1 Hacettepe Journal of Mathematics and Statistics

$\bigcap$ Volume 45 (5) (2016), 1329-1334

\title{
Asymptotic behavior of associated primes of certain ext modules
}

\author{
Kamal Bahmanpour*, Reza Naghipour ${ }^{\dagger}$ and Mehdi Sehatkhah ${ }^{\ddagger}$
}

\begin{abstract}
Let $R$ be a commutative Noetherian ring, $I$ an ideal of $R$ and $M$ a finitely generated $R$-module. It is shown that, whenever $I$ is principal, then for each integer $i$ the set of associated prime ideals $\operatorname{Ass}_{R} \operatorname{Ext}_{R}^{i}\left(R / I^{n}, M\right), n=1,2, \ldots$, becomes independent of $n$, for large $n$.
\end{abstract}

Keywords: Artinian module, Associated prime, Depth, Noetherian module. 2000 AMS Classification: 13E05.

Received : 20.07.2015 Accepted: 24.11.2015 Doi : 10.15672/HJMS.20164514288

\section{Introduction}

Let $R$ denote a commutative Noetherian ring (with identity), $I$ an ideal of $R$, and $M$ a finitely generated $R$-module. In [7] L.J. Ratliff, Jr., conjectured about the asymptotic behaviour of $\operatorname{Ass}_{R} R / I^{n}$ when $R$ is a Noetherian domain. Subsequently, M. Brodmann [1] showed that $\operatorname{Ass}_{R} M / I^{n} M$ is ultimately constant for large $n$. In [6], Melkersson and Schenzel asked whether the sets $\operatorname{Ass}_{R} \operatorname{Ext}_{R}^{i}\left(R / I^{n}, M\right)$ become stable for sufficiently large $n$. The aim of this paper is to show that, for all $i \geq 0$, the sets of prime ideals $\operatorname{Ass}_{R} \operatorname{Ext}_{R}^{i}\left(R / I^{n}, M\right), n=1,2, \ldots$, becomes independent of $n$, for large $n$, whenever $I$ is principal, which is an affirmative answer to the above question in the case $I$ is principal. Also, it is shown that, if $I$ is generated by an $R$-regular sequence and $\operatorname{Ext}_{R}^{i}(R / I, M)$ is Artinian, then the set $\cup_{n=1}^{\infty} \operatorname{Ass}_{R} \operatorname{Ext}_{R}^{i+1}\left(R / I^{n}, M\right)$ is finite.

For any $R$-module $L$, the set $\left\{\mathfrak{p} \in \operatorname{Ass}_{R} L \mid \operatorname{dim} R / \mathfrak{p}=\operatorname{dim} L\right\}$ is denoted by $\operatorname{Assh}_{R} L$.

*Department of Mathematics, Faculty of Sciences, University of Mohaghegh Ardabili, 5619911367, Ardabil, Iran.

Email : bahmanpour.k@gmail.com

${ }^{\dagger}$ Department of Mathematics, University of Tabriz, Tabriz, Iran.

Email : naghipour@ipm.ir

Email : naghipour@tabrizu.ac.ir Corresponding author.

${ }_{\ddagger}^{\ddagger}$ Roshdiyeh Higher Education Institution, Tabriz, Iran.

Email : m_sehhat@pnu.ac.ir 


\section{The Results}

2.1. Lemma. Let $R$ be a Noetherian ring, $I$ an ideal of $R$ and $M$ a finitely generated $R$-module. Then the sequence $\operatorname{Ass}_{R} \operatorname{Ext}_{R}^{1}\left(R / I^{n}, M\right)$ becomes eventually constant, for large $n$.

Proof. See [4, Corollary 2.3].

2.2. Lemma. Let $x$ be an element of the Noetherian ring $R$. Let $M$ and $N$ be two finitely generated $R$-modules such that $\operatorname{pd}(N)=t<\infty$. Then for each $i \geq t+2$ and for all large $k$,

$$
\operatorname{Ass}_{R} \operatorname{Ext}_{R}^{i}\left(N / x^{k} N, M\right)=\operatorname{Ass}_{R} \operatorname{Ext}_{R}^{i-1}\left(N / \Gamma_{R x}(N), M\right)
$$

and so the sets $\operatorname{Ass}_{R} \operatorname{Ext}_{R}^{i}\left(N / x^{k} N, M\right)$ are eventually constant.

Proof. Suppose that $i \geq t+2$. As, $N$ is finitely generated, it follows that there is an integer $n$ such that

$$
\Gamma_{R x}(N):=\bigcup_{i=0}^{\infty}\left(0:_{M} R x^{i}\right)=\left(0:_{N} x^{n}\right)=\left(0:_{N} x^{n+1}\right)=\cdots .
$$

Now we claim that for any $k \geq n$,

$$
\operatorname{Ext}_{R}^{i}\left(N / x^{k} N, M\right) \cong \operatorname{Ext}_{R}^{i-1}\left(N / \Gamma_{R x}(N), M\right) .
$$

To do this, as $\left(0:_{N} x^{k}\right)=\Gamma_{R x}(N)$, it follows that $x^{k} N \cong N / \Gamma_{R x}(N)$. Therefore for all $j \geq 0$ we have

$$
\operatorname{Ext}_{R}^{j}\left(x^{k} N, M\right) \cong \operatorname{Ext}_{R}^{j}\left(N / \Gamma_{R x}(N), M\right)
$$

for all $k \geq n$. Now the exact sequence

$$
0 \longrightarrow x^{k} N \longrightarrow N \longrightarrow N / x^{k} N \longrightarrow 0
$$

implies that

$$
\operatorname{Ext}_{R}^{i}\left(N / x^{k} N, M\right) \cong \operatorname{Ext}_{R}^{i-1}\left(x^{k} N, M\right) \cong \operatorname{Ext}_{R}^{i-1}\left(N / \Gamma_{R x}(N), M\right),
$$

(Note that $\operatorname{pd}(N)=t$ and $i \geq t+2$.) Hence we have

$$
\operatorname{Ass}_{R} \operatorname{Ext}_{R}^{i}\left(N / x^{k} N, M\right)=\operatorname{Ass}_{R} \operatorname{Ext}_{R}^{i-1}\left(N / \Gamma_{R x}(N), M\right),
$$

for all $k \geq n$, as required.

2.3. Theorem. Let $R$ be a Noetherian ring and let $x$ be an element of $R$. Let $M$ be a finitely generated $R$-module and $i$ a non-negative integer. Then the sequence

$$
\operatorname{Ass}_{R} \operatorname{Ext}_{R}^{i}\left(R / R x^{k}, M\right),
$$

of associated primes is ultimately constant for large $k$, and if $i \geq 2$, then

$$
\operatorname{Ass}_{R} \operatorname{Ext}_{R}^{i}\left(R / R x^{k}, M\right)=\operatorname{Ass}_{R} \operatorname{Ext}_{R}^{i-1}\left(R / \Gamma_{R x}(R), M\right),
$$

for all large $k$.

Proof. The result follows from Lemmas 2.1 and 2.2.

2.4. Proposition. Let $R$ be a Noetherian ring and let $M, N$ be tow finitely generated $R$-modules. Let $x$ be an $N$-regular element of $R$. Then, for any given integer $j \geq 0$, the set

$$
\bigcup_{n=1}^{\infty} \operatorname{Ass}_{R} \operatorname{Ext}_{R}^{j}\left(N / x^{n} N, M\right),
$$

of associated prime ideals, is finite. 
Proof. If $j=0$, then

$$
\operatorname{Ass}_{R} \operatorname{Hom}_{R}\left(N / x^{n} N, M\right)=\operatorname{Ass}_{R} \operatorname{Hom}_{R}\left(N, \operatorname{Hom}_{R}(R / R x, M)\right),
$$

and so

$$
\bigcup_{n=1}^{\infty} \operatorname{Ass}_{R} \operatorname{Ext}_{R}^{0}\left(N / x^{n} N, M\right)
$$

is a finite set. Suppose then that $j \geq 1$, and we use the exact sequence

$$
0 \longrightarrow N \stackrel{x^{n}}{\longrightarrow} N \longrightarrow N / x^{n} N \longrightarrow 0,
$$

to obtain the exact sequence

$$
\begin{gathered}
\cdots \longrightarrow \operatorname{Ext}_{R}^{j-1}(N, M) \stackrel{x^{n}}{\longrightarrow} \operatorname{Ext}_{R}^{j-1}(N, M) \longrightarrow \operatorname{Ext}_{R}^{j}\left(N / x^{n} N, M\right) \\
\longrightarrow \operatorname{Ext}_{R}^{j}(N, M) \stackrel{x^{n}}{\longrightarrow} \operatorname{Ext}_{R}^{j}(N, M) \longrightarrow \cdots .
\end{gathered}
$$

Hence we have the following exact sequence,

$$
0 \rightarrow \operatorname{Ext}_{R}^{j-1}(N, M) / x^{n} \operatorname{Ext}_{R}^{j-1}(N, M) \rightarrow \operatorname{Ext}_{R}^{j}\left(N / x^{n} N, M\right) \rightarrow\left(0:_{\operatorname{Ext}_{R}^{j}(N, M)} x^{n}\right) \rightarrow 0 .
$$

Consequently, it follows from Brodmann's result (see [1]) that the set

$$
\bigcup_{n=1}^{\infty} \operatorname{Ass}_{R} \operatorname{Ext}_{R}^{j}\left(N / x^{n} N, M\right)
$$

is finite.

2.5. Lemma. Let $R$ be a Noetherian ring and let $M$ be an $R$-module. Let $N$ be an Artinian submodule of $M$. Then

$$
\operatorname{Ass}_{R} M / N \backslash \operatorname{Supp} N=\operatorname{Ass}_{R} M \backslash \operatorname{Supp} N .
$$

Proof. As $N$ is an Artinian $R$-module, it follows that the set $\operatorname{Supp} N \subseteq \operatorname{Max} R$ is finite. Let $\operatorname{Supp} N=\left\{\mathfrak{m}_{1}, \ldots, \mathfrak{m}_{n}\right\}$ and $J:=\mathfrak{m}_{1} \cdots \mathfrak{m}_{n}$. Then we have

$$
\operatorname{Ass}_{R} M \backslash \operatorname{Supp} N=\operatorname{Ass}_{R} M / \Gamma_{J}(M)=\operatorname{Ass}_{R} M / N \backslash \operatorname{Supp} N,
$$

as required.

Following we let $H_{I}^{j}(M)$ denote the $j^{\text {th }}$ local cohomology module of $M$ with respect to an ideal $I$ of a Noetherian ring $R$ (cf. [2] and [3]).

2.6. Theorem. Let $R$ be a Noetherian ring and let $I$ be an ideal of $R$ which is generated by an $R$-regular sequence. Let $M$ be a finitely generated $R$-module and let $i$ be a nonnegative integer such that the $R$-module $\operatorname{Ext}_{R}^{i}(R / I, M)$ is Artinian. Then the set

$$
\bigcup_{n=1}^{\infty} \operatorname{Ass}_{R} \operatorname{Ext}_{R}^{i+1}\left(R / I^{n}, M\right),
$$

is finite. In particular, the set $\operatorname{Ass}_{R} H_{I}^{i+1}(M)$ is finite.

Proof. For $n \geq 0$, the exact sequence

$$
0 \longrightarrow I^{n} / I^{n+1} \longrightarrow R / I^{n+1} \longrightarrow R / I^{n} \rightarrow 0
$$

induces the exact sequence

$\operatorname{Ext}_{R}^{i}\left(I^{n} / I^{n+1}, M\right) \rightarrow \operatorname{Ext}_{R}^{i+1}\left(R / I^{n}, M\right) \rightarrow \operatorname{Ext}_{R}^{i+1}\left(R / I^{n+1}, M\right) \rightarrow \operatorname{Ext}_{R}^{i+1}\left(I^{n} / I^{n+1}, M\right)$. 
Since $I$ is generated by an $R$-regular sequence, by [5, page 125] $I^{n} / I^{n+1}$ is a finitely generated free $R / I$-module, and so the sets

$$
\begin{gathered}
\operatorname{Ass}_{R} \operatorname{Ext}_{R}^{i+1}\left(I^{n} / I^{n+1}, M\right)=\operatorname{Ass}_{R} \operatorname{Ext}_{R}^{i+1}(R / I, M), \text { and } \\
\operatorname{SuppExt}_{R}^{i}\left(I^{n} / I^{n+1}, M\right)=\operatorname{SuppExt}_{R}^{i}(R / I, M)
\end{gathered}
$$

are finite, (note that the $R$-module $\operatorname{Ext}_{R}^{i}(R / I, M)$ is Artinian). Therefore in view of the above exact sequence and Lemma 2.5 , the set

$$
\operatorname{Ass}_{R} \operatorname{Ext}_{R}^{i+1}\left(R / I^{n+1}, M\right) \backslash \operatorname{SuppExt}_{R}^{i}(R / I, M)
$$

is a subset of

$$
\left(\operatorname{Ass}_{R} \operatorname{Ext}_{R}^{i+1}\left(R / I^{n}, M\right) \backslash \operatorname{SuppExt}_{R}^{i}(R / I, M)\right) \cup \operatorname{Ass}_{R} \operatorname{Ext}_{R}^{i+1}(R / I, M),
$$

and so the set $\bigcup_{n=1}^{\infty} \operatorname{Ass}_{R} \operatorname{Ext}_{R}^{i+1}\left(R / I^{n}, M\right)$ is finite, as required. The second assertion follows from the fact that

$$
\operatorname{Ass}_{R} H_{I}^{i+1}(M) \subseteq \bigcup_{n=1}^{\infty} \operatorname{Ass}_{R} \operatorname{Ext}_{R}^{i+1}\left(R / I^{n}, M\right) .
$$

2.7. Corollary. Let $R$ be a Noetherian ring and let $I$ be an ideal of $R$ which is generated by an $R$-regular sequence. Let $M$ be a finitely generated $R$-module and let $i$ be a nonnegative integer such that $\operatorname{Ext}_{R}^{i}(R / I, M)=0$. Then the sequence

$$
\operatorname{Ass}_{R} \operatorname{Ext}_{R}^{i+1}\left(R / I^{k}, M\right),
$$

of associated primes is increasing and ultimately constant for all large $k$.

Proof. Since $I^{k} / I^{k+1}$ is a free $R / I$-module, it follows that $\operatorname{Ext}_{R}^{i}\left(I^{k} / I^{k+1}, M\right)=0$, for all $k \geq 1$. Hence the exact sequence

$$
0 \longrightarrow \operatorname{Ext}_{R}^{i+1}\left(R / I^{k}, M\right) \longrightarrow \operatorname{Ext}_{R}^{i+1}\left(R / I^{k+1}, M\right) \longrightarrow \operatorname{Ext}_{R}^{i+1}\left(I^{k} / I^{k+1}, M\right),
$$

implies that

$$
\operatorname{Ass}_{R} \operatorname{Ext}_{R}^{i+1}\left(R / I^{k}, M\right) \subseteq \operatorname{Ass}_{R} \operatorname{Ext}_{R}^{i+1}\left(R / I^{k+1}, M\right) .
$$

Now the result follows from Theorem 2.6.

2.8. Lemma. Let $(R, \mathfrak{m})$ be a Noetherian local ring of depth $d$. Let $M$ be a finitely generated $R$-module and $N$ an Artinan submodule of $M$. Then for all $i \leq d-1$,

$$
\operatorname{Ext}_{R}^{i}(M, R) \cong \operatorname{Ext}_{R}^{i}(M / N, R) \text {. }
$$

Proof. The exact sequence

$$
0 \longrightarrow N \longrightarrow M \longrightarrow M / N \longrightarrow 0
$$

induces the exact sequence

$$
\operatorname{Ext}_{R}^{i-1}(N, R) \longrightarrow \operatorname{Ext}_{R}^{i}(M / N, R) \longrightarrow \operatorname{Ext}_{R}^{i}(M, R) \longrightarrow \operatorname{Ext}_{R}^{i}(N, R) .
$$

As $N$ has finite length and $\operatorname{depth} R=d$, it follows that

$$
\operatorname{Ext}_{R}^{i}(N, R)=0=\operatorname{Ext}_{R}^{i-1}(N, R) .
$$

Hence the result follows.

2.9. Lemma. Let $(R, \mathfrak{m})$ be a local Cohen-Macaulay ring of dimension $d$ and $I$ an ideal of $R$. Then for any $\mathfrak{p} \in \operatorname{Ass}_{R} \operatorname{Ext}_{R}^{\text {grade } I}(R / I, R)$,

$$
\text { height } \mathfrak{p}=\text { grade } I \text {. }
$$


Proof. Let grade $I=t$. The assertion is clear when $t=0$. Now suppose that, $t \geq 1$. There exists an $R$-regular sequence $x_{1}, \ldots, x_{t} \in I$. As

$$
\operatorname{Ext}_{R}^{\text {grade } I}(R / I, R) \cong \operatorname{Hom}_{R /\left(x_{1}, \ldots, x_{t}\right)}\left(R / I, R /\left(x_{1}, \ldots, x_{t}\right)\right)
$$

and $R /\left(x_{1}, \ldots, x_{t}\right)$ is a Cohen-Macaulay ring it follows that

$$
\operatorname{Ass}_{R} \operatorname{Ext}_{R}^{\text {grade } I}(R / I, R) \subseteq \operatorname{Assh}_{R} R /\left(x_{1}, \ldots, x_{t}\right),
$$

that implies for any $\mathfrak{p} \in \operatorname{Ass}_{R} \operatorname{Ext}_{R}^{\text {grade } I}(R / I, R)$,

$$
\text { height } \mathfrak{p}=\operatorname{grade} I \text {, }
$$

as required.

2.10. Theorem. Let $(R, \mathfrak{m})$ be a local Cohen-Macaulay ring of dimension $d \geq 3$. Let $I$ be an ideal of $R$ such that $1 \leq \operatorname{grade} I \leq d-2$. Then

$$
\operatorname{depth} \operatorname{Ext}_{R}^{\text {grade } I}(R / I, R) \geq 2,
$$

and if grade $I \leq d-3$ then the equality holds if and only if $\mathfrak{m} \in \operatorname{Ass}_{R} \operatorname{Ext}_{R}^{1+\operatorname{grade} I}(R / I, R)$.

Proof. Set $t:=\operatorname{grade} I$. Let $\Gamma_{\mathfrak{m}}(R / I):=J / I$ for some ideal $J$ of $R$ with $I \subseteq J$. Then it is easy to see that $\mathfrak{m} \notin \operatorname{Ass}_{R} R / J$ and $\operatorname{dim} R / I=\operatorname{dim} R / J$. Hence as $R$ is a Cohen-Macaulay ring, it follows that grade $I=$ grade $J$. Moreover, since $J / I$ has finite length, it follows from Lemma 2.8 that

$$
\operatorname{Ext}_{R}^{t}(R / I, R) \cong \operatorname{Ext}_{R}^{t}(R / J, R) \text { and } \operatorname{Ext}_{R}^{t+1}(R / I, R) \cong \operatorname{Ext}_{R}^{t+1}(R / J, R) .
$$

Therefore, we may and do replace $I$ with $J$ in the following. Since $\mathfrak{m} \notin \operatorname{Ass}_{R} R / J$, it follows that there exists an element $x \in R$ such that $x$ is $R / J$-regular sequence. Then, as $\operatorname{dim} R /(J+R x)=\operatorname{dim} R / J-1$ and $R$ is a Cohen-Macaulay ring, it follows that

$$
\operatorname{grade}(J+R x)=\operatorname{grade} J+1 \text {. }
$$

Now the exact sequence

$$
0 \rightarrow R / J \stackrel{x}{\rightarrow} R / J \rightarrow R / J+R x \rightarrow 0
$$

induces the exact sequence

$$
0 \rightarrow \operatorname{Ext}_{R}^{t}(R / J, R) \stackrel{x}{\rightarrow} \operatorname{Ext}_{R}^{t}(R / J, R) \rightarrow \operatorname{Ext}_{R}^{t+1}(R / J+R x, R) .
$$

Hence

$$
\operatorname{Ass}_{R} \operatorname{Ext}_{R}^{t}(R / J, R) / x \operatorname{Ext}_{R}^{t}(R / J, R) \subseteq \operatorname{Ass}_{R} \operatorname{Ext}_{R}^{t+1}(R / J+R x, R),
$$

and since $1+$ grade $J \leq d-1$, it follows from Lemma 2.9 that

$$
\mathfrak{m} \notin \operatorname{Ass}_{R} \operatorname{Ext}_{R}^{t+1}(R / J+R x, R) .
$$

Now, it easily follows that

$$
\operatorname{depth} \operatorname{Ext}_{R}^{t}(R / J, R) \geq 2 .
$$

Now, let grade $J \leq d-3$. Then we have the following exact sequence, $0 \rightarrow \operatorname{Ext}_{R}^{t}(R / J, R) / x \operatorname{Ext}_{R}^{t}(R / J, R) \rightarrow \operatorname{Ext}_{R}^{t+1}(R / J+R x, R) \rightarrow\left(0:_{\operatorname{Ext}_{R}^{t+1}(R / J, R)} x\right) \rightarrow 0$. Since grade $(J+R x)=t+1$ and $t+1 \leq d-2$, it follows from the first part that $\operatorname{depth} \operatorname{Ext}_{R}^{t+1}(R / J+R x, R) \geq 2$. Therefore it follows from the exact sequence

$0 \rightarrow \operatorname{Hom}_{R}\left(R / \mathfrak{m},\left(0:_{\operatorname{Ext}_{R}^{t+1}(R / J, R)} x\right)\right) \rightarrow \operatorname{Ext}_{R}^{1}\left(R / \mathfrak{m}, \operatorname{Ext}_{R}^{t}(R / J, R) / x \operatorname{Ext}_{R}^{t}(R / J, R)\right) \rightarrow 0$ that depth $\operatorname{Ext}_{R}^{t}(R / J, R)=2$ if and only if $\operatorname{Hom}_{R}\left(R / \mathfrak{m},\left(0:_{\operatorname{Ext}_{R}^{t+1}(R / J, R)} x\right)\right) \neq 0$. Consequently depth $\operatorname{Ext}_{R}^{t}(R / J, R)=2$ if and only if $\mathfrak{m} \in \operatorname{Ass}_{R} \operatorname{Ext}_{R}^{t+1}(R / J, R)$, as required. 


\section{Acknowledgments}

The authors are deeply grateful to the referee for his/her valuable suggestions on the article. Also, we would like to thank Professor Hossein Zakeri for his reading of the first draft and useful comments.

\section{References}

[1] M. Brodmann, Asymptotic stability of $\operatorname{Ass}\left(M / I^{n} M\right)$, Proc. Amer. Math. Soc. 74(1979), 16-18.

[2] M.P. Brodmann and R.Y. Sharp, Local cohomology; an algebraic introduction with geometric applications, Cambridge University Press, Cambridge, 2013.

[3] A. Grothendieck, Local cohomology, Notes by R. Hartshorne, Lecture Notes in Math., 862 (Springer, New York, 1966).

[4] K. Khashyarmanesh and S. Salarian, Asymptotic stability of $\operatorname{Att}_{R} \operatorname{Tor}_{1}^{R}\left(R / \mathfrak{a}^{n}, A\right)$, Proc. Edin. Math. Soc. 44(2001), 479-483.

[5] H. Matsumura, Commutative ring theory, Cambridge Univ. Press, Cambridge, UK, 1986.

[6] L. Melkersson and P. Schenzel, Asymptotic prime ideals related to derived functors, Proc. Amer. Math. Soc.117 (1993), 935-938.

[7] L.J. Ratliff, Jr., On the prime divisors of $I^{n}, n$ large, Michigan Math. J. 23 (1976), 337-352. 